\title{
A new development in quantum field equations of dyons
}

\author{
B. C. Chanyal \\ Department of Physics, \\ G. B. Pant University of Agriculture \&5 Technology, \\ Pantnagar-263145 (Uttarakhand), India \\ E-mail: bcchanyal@gmail.com,bcchanyal@gbpuat.ac.in
}

\begin{abstract}
In this study, we describe a novel approach to quantum phenomena of the generalized electromagnetic fields of dyons with quaternionic analysis. Starting with quaternionic quantum wave equations, we have established a quantized condition for time coordinate that transforms microscopic to macroscopic fields. In view of classical electromagnetic field equation, we propose a new set of quantized Proca-Maxwell's equations for dyons. Furthermore, a quantized form of four-currents densities and the quantized Lorentz gauge conditions, respectively for electric and magnetic potentials of dyons are obtained. We have established the new quantized continuity equations for electric and magnetic densities of dyons which associated with a torque density result from the two spin states. The quantized Klein-Gordon like field equations and the unified quaternionic electromagnetic potential wave equations for massive dyons are demonstrated. Moreover, we investigate the quaternionic quantized relativistic Dirac field equations for massive dyons, which indicated that there will be the existence of antiparticle of dyons called antidyons.
\end{abstract}

Keywords: quaternion, dyons and anti-dyons, Maxwell equations, quantized Lorentz gauge, Dirac field equation,

PACS: 02.10.De, 03.50.De, 03.65.-w

\section{Introduction}

The generalized Maxwell's equations describe a complete and valuable description to the classical behavior of electric and magnetic fields. At the end of the twentieth-century, the asymmetry between electric and magnetic field equations became very clear with the formulation of Maxwell's equations. In 1931, Dirac [1, 2] investigated a new particle called 'magnetic monopoles' and also generalized the usual electrodynamics. The basic idea for magnetic monopoles is that, it will be stable particles, which carry magnetic charges like electric charges for electrons, ought to exist has proved to be remarkably durable. The existence of particles with the magnetic charge (or monopole) [1, 2] implies that the electric charge must be integer multiples of a fundamental units. Beside, a quantization of electric charges have been actually observed in nature. Dirac also have confirmed the constancy of quantum physics with unique Dirac equation, particularly in solving the difficulties associated with the string singularity identified by Dirac [2, 3]. Thus, we can say that the magnetic monopole would symmeterize in Maxwell's equations, but there would be some numerical asymmetry. These types of discrepancy could be the basis for the introduction of what we may call the 'classical magnetic 
monopole'. In this classical formulation there was no prediction for the magnetic-monopole mass. Rather, a kind of rule was established, and also assumed that the radius of classical electron may be equal to the radius of classical monopole from which one has $m_{0}^{g}=\mathrm{g}_{d}^{2} m_{0}^{e} / e^{2} \approx 4700 m_{0}^{e} \approx 2.4 G e V$ (approx). This ingenious suggestions with the existence of magnetic-monopole gave rise to considerable literature [4]-[10] that subjected to predict the mass, size, spin, parity and other quantum properties of monopoles. In the case of the existence of magnetic monopole, Schwinger [11-[13] formulated a novel relativistic quantum field theory of magnetic charges which maintained the complete symmetry between electric and magnetic fields. The Dirac's quantization condition governed the product of electric and magnetic charge to integer values. The combined theories of Schwinger [11]-[13] and Zwanziger [14] identify the theory of dyons (i.e., particles carrying simultaneous existence of electric and magnetic charges). The quantized theory, though explains to some extent the negative experimental results in search of magnetic monopole, and required to maintained the rotational symmetry which was violated due to existence of singular lines which comes from the solution of vector potential around a monopole. Peres [15] discussed the controversial nature [16] of these singular lines of monopole [1, 17 and then the charged quantization condition has taken place in purely group theoretical manner. For electromagnetic fields, Maxwell equations are considered to be the classical description and the quantization of these classical fields gives a relativistic covariant quantum description. Furthermore, Bialynicki-Birula [18, 19] and Sipe [20] independently explained that Maxwell equations are the quantum description of electromagnetic fields at the first quantum level and the Fermat principle for light forms the classical description of electromagnetic fields similar to the Hamilton's variational principle for particles with mass.

In quaternionic space-time, Rajput et. al. 21] proposed a unified theory of generalized electromagnetic and gravitational fields associated with massive dyons. Many other authors [22, 23] discussed the development of classical and quantum theory of electrodynamics in case of experimental data. Chanyal et al. [24]29] have studied classical field equations and conservation laws of dyons in terms of octonion algebra and explained the corresponding generalized Dirac Maxwell's equations with the equation of motion in compact manner. Moreover, Arbab [30, 31] derived the role of quaternionic quantum mechanics in case of quantized Maxwell equations. Besides, in literature [32- 41, the reformulation of the field equations of magnetic monopoles and massive dyons have been discussed in terms of hyper complex numbers including quaternions, octonions and sedenions. Recently, Chanyal 42 independently proposed a covariant theory of relativistic quantum mechanics for dyons wave propagation in terms of quaternionic formalism. Keeping in mind the recent work in quaternionic quantum mechanics, we establish a novel approach to quantum phenomena in the generalized electromagnetic fields of dyons with quaternionic analysis. Starting with quaternionic four dimensional structure and its properties, we propose the relativistic quantized wave equations. Since classical theory is a approximation of quantum theory, in this study, we establish a quantized condition for time coordinate that transforms quantum to classical field. We describe a compact set of quantized ProcaMaxwell's equations for dyons. Furthermore, a quantized form of four potentials, four-currents densities and also the quantized Lorentz gauge conditions, respectively for electric and magnetic potentials of dyons are obtained. We obtain a new quantized continuity equation for generalized electric and magnetic four-densities of dyons which associated with a torque density result from the two spin states. The quantized Klein-Gordon like field equations and the unified quaternionic electromagnetic potential wave equations for massive dyons are also obtained. Moreover, we investigate the quaternionic quantized relativistic Dirac field equations for massive dyons, which indicated that there will be the existence of antiparticle of dyons called antidyons. Our quaternionic quantum theory will help to establish the existence for various massive particles. 


\section{The quaternion}

In mathematics, a geometric algebra is the largest possible associative division algebra that integrates all algebraic systems (viz., algebra of complex numbers, vector algebra, matrix algebra, quaternion algebra, etc.) into a coherent mathematical language. Interestingly, after the real and the complex algebras, quaternion 43 is first hyper-complex division algebra. It has more significant impacts on mathematics and theoretical physics. The quaternion algebra (called q-algebra), defined by $\mathbb{Q}$, is a four-dimensional algebra [43, 44] over the field of real numbers. A real quaternion $\mathbb{Q}$ and its quaternionic conjugate $\overline{\mathbb{Q}}$ may be expressed as

$$
\begin{aligned}
& \mathbb{Q}=\left(q_{0}, \vec{q}\right) \equiv q_{0} e_{0}+\sum_{j=1}^{3} q_{j} e_{j}, \quad(\forall j=1,2,3) \\
& \overline{\mathbb{Q}}=\left(q_{0},-\vec{q}\right) \equiv q_{0} \bar{e}_{0}+\sum_{j=1}^{3} q_{j} \bar{e}_{j}=q_{0} e_{0}-\sum_{j=1}^{3} q_{j} e_{j},
\end{aligned}
$$

where $\left(q_{0} e_{0}\right)$ and $\left(q_{j} e_{j}\right) \forall j=1,2,3$ are the scalar and vector parts of q-algebra. The q-units defined by $\left(e_{0}, e_{1}, e_{2}, e_{3}\right)$ are known as the quaternion basis. The properties of q-algebra are given by:

$$
\begin{aligned}
e_{0}^{2} & =e_{0}=1, e_{j}^{2}=-e_{0}, \bar{e}_{j}=-e_{j}, \bar{e}_{0}=e_{0} \\
e_{0} e_{j} & =e_{j} e_{0}=e_{j}, e_{i} e_{j}=-\delta_{i j}+\varepsilon_{i j k} e_{k} \quad(\forall i, j, k=1,2,3) .
\end{aligned}
$$

In equation (2.3) we have used $\delta_{i j}$ as delta symbol, $\bar{e}_{j}$ as quaternionic conjugate basis and $\varepsilon_{i j k}$ as the Levi Civita three index symbol. Since, the q-algebra is an associative as well as non-commutative algebra in nature and holds the usual distribution law, i.e., $\left(e_{j} e_{k}\right) e_{l}=e_{j}\left(e_{k} e_{l}\right) \forall i, j, k=1,2,3$. Moreover, we may distinguish the real part of the quaternion $q_{0}$ as,

$$
\mathfrak{R e} \mathbb{Q}=\frac{1}{2}(\overline{\mathbb{Q}}+\mathbb{Q})=q_{0} .
$$

If $\mathfrak{R e} \mathbb{Q}=0$ or $q_{0}=0$, then we may found the imaginary part of the q-algebra and the condition become $\mathbb{Q}=-\overline{\mathbb{Q}}$, where

$$
\mathfrak{I m} \mathbb{Q}=\frac{1}{2}(\mathbb{Q}-\overline{\mathbb{Q}})=\left(q_{1} e_{1}+q_{2} e_{2}+q_{3} e_{3}\right)
$$

Furthermore, the summation and the multiplication of any two quaternionic variables are given by

$$
\begin{aligned}
\mathbb{P} \pm \mathbb{Q} & =\left(p_{0} \pm q_{0}\right)+(\vec{p} \pm \vec{q}) \\
& =\left(p_{0} \pm q_{0}\right) e_{0}+\left(p_{1} \pm q_{1}\right) e_{1}+\left(p_{1} \pm q_{1}\right) e_{2}+\left(p_{1} \pm q_{1}\right) e_{3}, \\
\mathbb{P} \mathbb{Q} & =\left[p_{0}+\vec{p}\right]\left[q_{0}+\vec{q}\right]=p_{0} q_{0}+p_{0} \vec{q}+q_{0} \vec{p}-(\vec{p} \cdot \vec{q})+(\vec{p} \times \vec{q}),
\end{aligned}
$$

where $(\cdot)$ and $(\times)$ are indicated dot and cross products like the usual three-dimensional scalar and vector products. In equation (2.7), we should notice that the q-multiplication $\mathbb{P} \mathbb{Q} \neq \mathbb{Q P}$, because $\vec{p} \times \vec{q} \neq-\vec{q} \times \vec{p}$. Rather, the norm of a q-algebra become $N(\mathbb{Q})=\mathbb{Q} \overline{\mathbb{Q}}=\overline{\mathbb{Q}} \mathbb{Q}=\sum_{\alpha=0}^{3} \mathbb{Q}_{\alpha}^{2}$, and the inverse of a q-algebra (non-zero norm) become $\mathbb{Q}^{-1}=\frac{\overline{\mathbb{Q}}}{N(\mathbb{Q})} ; \mathbb{Q} \mathbb{Q}^{-1}=\mathbb{Q}^{-1} \mathbb{Q}=1$. The norm of a q-algebra is zero if $\mathbb{Q}=0$, and is always positive otherwise. Thus keeping in mind the q-properties, in the next sections we shall implement 
q-algebra in relativistic quantum mechanics.

\section{Quaternion approach to relativistic quantum field equations}

In physics point of view, the four independent quantities such as the four-position, four-momentum, four-force, four-potential and four-current etc. are suitable to express by q-algebra. In other words, the quaternionic approach is a such type of novel approach where we generalize and explain beautifully to the quantum behavior of relativistic equations. In order to discuss the quaternionic quantum mechanics (qQM), we may start with the momentum eigen value equation [30, 31,

$$
\breve{P} \breve{\Psi}=\Upsilon \breve{\Psi}, \quad(\breve{P}, \breve{\Psi} \in \mathbb{Q})
$$

where $\Upsilon \sim\left(m_{0} c\right)$ is the eigen value of quaternionic four-momentum operator $\breve{P}=\left\{\vec{p}, \frac{i}{c} E\right\}$, the eigen

function $\breve{\Psi}=\left\{\vec{\Psi}, \frac{i}{c} \Psi_{0}\right\}$ is quaternionic four-wave function, $m_{0}$ is the mass of particle (dyon) and $c$ is the speed of light. By using the quaternionic product for two variables given by (2.7) the quaternionic momentum eigen value equation (3.1) leads to

$$
\underbrace{\left(\frac{i}{c} E\right) \vec{\Psi}+\vec{p}\left(\frac{i}{c} \Psi_{0}\right)+\vec{p} \times \vec{\Psi}}_{\text {vector part }}, \underbrace{-\frac{E}{c^{2}} \Psi_{0}-\vec{p} \cdot \vec{\Psi}}_{\text {scalar part }}=m_{0} c\left\{\vec{\Psi}, \frac{i}{c} \Psi_{0}\right\} .
$$

Now, the quantum equations for a moving particle can be expressed by [45, 46,

$$
\begin{aligned}
\vec{\nabla} \cdot \vec{\Psi}-\frac{1}{c^{2}} \frac{\partial \Psi_{0}}{\partial t}-\frac{m_{0}}{\hbar} \Psi_{0} & =0, \\
\vec{\nabla} \Psi_{0}-\frac{\partial \vec{\Psi}}{\partial t}-\frac{m_{0} c^{2}}{\hbar} \vec{\Psi} & =0 \\
\vec{\nabla} \times \vec{\Psi} & =0
\end{aligned}
$$

where the momentum and energy operator are $\vec{p}=-i \hbar \vec{\nabla}$ and $E=i \hbar \frac{\partial}{\partial t}$. The beauty of the equation (3.1) is that, it exhibited the well-known Dirac equation for massive dyons, i.e., $\left(i \hbar \gamma^{\nu} \partial_{\nu}-m_{0} c\right) \breve{\Psi}=0$ if we put the four dimensional quaternionic momentum operator $\breve{P} \longrightarrow\left(i \hbar \gamma^{\nu} \partial_{\nu}\right)$. We may established the second order quantum differential equations by operating $\vec{\nabla}$ to the both side from left on equations (3.3] 3.5), i.e.

$$
\begin{gathered}
\nabla^{2} \vec{\Psi}-\frac{1}{c^{2}} \frac{\partial^{2} \vec{\Psi}}{\partial t^{2}}-2\left(\frac{m_{0}}{\hbar}\right) \frac{\partial \vec{\Psi}}{\partial t}-\frac{m_{0}^{2} c^{2}}{\hbar^{2}} \vec{\Psi}=0, \\
\nabla^{2} \Psi_{0}-\frac{1}{c^{2}} \frac{\partial^{2} \Psi_{0}}{\partial t^{2}}-2\left(\frac{m_{0}}{\hbar}\right) \frac{\partial \Psi_{0}}{\partial t}-\frac{m_{0}^{2} c^{2}}{\hbar^{2}} \Psi_{0}=0 \\
\vec{\nabla} \times(\vec{\nabla} \times \vec{\Psi}) \equiv \vec{\nabla}(\vec{\nabla} \cdot \vec{\Psi})-\nabla^{2} \vec{\Psi}=0
\end{gathered}
$$

Equation (3.6) represents the generalized quaternionic quantum wave equation for massive vector field $(\vec{\Psi})$ which may visualized by Dirac field, whereas equation (3.7) represents to massive scalar field (like as the Klein-Gordon field $\Psi_{0}$ ). Equation (3.8) shows the quaternionic condition for the triple vector multiplication. 
The unified structure of quaternionic quantum wave equation is expressed by

$$
\nabla^{2} \breve{\Psi}-\frac{1}{c^{2}} \frac{\partial^{2} \breve{\Psi}}{\partial t^{2}}-2\left(\frac{m_{0}}{\hbar}\right) \frac{\partial \breve{\Psi}}{\partial t}-\frac{m_{0}^{2} c^{2}}{\hbar^{2}} \breve{\Psi}=0
$$

Here we should notice that, in quaternionic quantum wave equation the additional term $\left(\frac{2 m_{0}}{\hbar}\right) \frac{\partial \breve{\Psi}}{\partial t}$ describes the time dependent source called the damping terms resulting from the inertia of the massive particle. A beauty of quaternionic unified wave equation is that we may transform this quantum wave equation into classical wave equation if we eliminate the damping term by introducing a new time coordinate $\tau$ as

$$
\frac{\partial}{\partial \tau} \longmapsto\left(\frac{\partial}{\partial t}+\frac{1}{\triangle \mathfrak{t}}\right), \text { where } \triangle \mathfrak{t}=\frac{\hbar}{m_{0} c^{2}} \approx \frac{\hbar}{\triangle E_{k}}
$$

This is the necessary condition for to transform macroscopic to microscopic field in quaternionic space-time. Then the ordinary macroscopic wave equations becomes,

$$
\begin{aligned}
& \nabla^{2} \vec{\Psi}-\frac{1}{c^{2}} \frac{\partial^{2} \vec{\Psi}}{\partial \tau^{2}}=0, \quad \Longrightarrow \diamond \vec{\Psi}=0 \\
& \nabla^{2} \Psi_{0}-\frac{1}{c^{2}} \frac{\partial^{2} \Psi_{0}}{\partial \tau^{2}}=0, \quad \Longrightarrow \diamond \Psi_{0}=0
\end{aligned}
$$

where $\diamond=\left(\nabla^{2}-\frac{1}{c^{2}} \frac{\partial^{2}}{\partial \tau^{2}}\right)$ denotes classical D' Alembert operator for time coordinate $\tau$.

Now, in view of the physical significant of generalized quantum wave equation, we may consider a general plane wave solution of equation (3.9) as

$$
\breve{\Psi}=\xi \exp i(\omega t-\vec{k} \cdot \vec{r}), \text { where } \xi=\text { constant. }
$$

By substituting (3.13) in equation (3.9), we may found two Dirac like solutions respectively for positive energy with particle energy $\hbar \omega^{+}$and negative energy with antiparticle energy $\hbar \omega^{-}$[2]. Furthermore, the group and phase velocities respectively, $v_{g}$ and $v_{p}$ can be expressed by the relations $v_{g}=\frac{\partial \omega^{ \pm}}{\partial k}= \pm c$, and $v_{p}=\frac{\omega^{ \pm}}{\vec{k}}=\frac{i m_{0} c^{2}}{\hbar \vec{k}} \pm c$.

\section{Quaternionic classical electromagnetic field of dyons}

In order to write the classical electromagnetic field of dyons [47, let us start with the quaternionic four-vector representation with an imaginary fourth component of Euclidean structure $(+,+,+,-)$. The quaternionic four dimensional world vector $\mathbb{X}$ and the four gradiant $\mathbb{D}$ may then be defined as

$$
\begin{aligned}
& \mathbb{X}\left(e_{1}, e_{2}, e_{3}, e_{0}\right):=\{x, y, z,-i c t\} \\
& \mathbb{D}\left(e_{1}, e_{2}, e_{3}, e_{0}\right):=\left\{\frac{\partial}{\partial x}, \frac{\partial}{\partial y}, \frac{\partial}{\partial z},-\frac{\partial}{i \partial(c t)}\right\} .
\end{aligned}
$$

As such, we may write a quaternionic four-vector $\mathbb{X}^{\nu}$ and its quaternion conjugate $\overline{\mathbb{X}^{\nu}}$ in terms of following covariant form,

$$
\begin{aligned}
& \mathbb{X}^{\nu}=\left(x^{0} e_{0}+x^{1} e_{1}+x^{2} e_{2}+x^{3} e_{3}\right), \\
& \overline{\mathbb{X}}^{\nu}=\left(x^{0} e_{0}-x^{1} e_{1}-x^{2} e_{2}-x^{3} e_{3}\right), \quad\left(\mathbb{X}^{\nu}, \overline{\mathbb{X}}^{\nu} \in \mathbb{Q}\right),
\end{aligned}
$$


and the quaternionic equation of spherical surface is governed as

$$
\sum_{\nu=0}^{3} \mathbb{X}^{\nu} \overline{\mathbb{X}}_{\nu} \equiv\left(x^{0} x_{0}+x^{1} x_{1}+x^{2} x_{2}+x^{3} x_{3}\right) \simeq-c^{2} t^{2}+x^{2}+y^{2}+z^{2} .
$$

Similarly, the classical D' Alembert operator defined by $\square$, can be expressed as

$$
\begin{aligned}
\square=\mathbb{D} \overline{\mathbb{D}}=\overline{\mathbb{D}} \mathbb{D} & \equiv \frac{\partial^{2}}{\partial x^{2}}+\frac{\partial^{2}}{\partial y^{2}}+\frac{\partial^{2}}{\partial z^{2}}-\frac{1}{c^{2}} \frac{\partial^{2}}{\partial t^{2}} \\
& =\left(\nabla^{2}-\frac{1}{c^{2}} \frac{\partial^{2}}{\partial t^{2}}\right),
\end{aligned}
$$

where $\overline{\mathbb{D}}$ is the quaternionic conjugate of the differential operator $\mathbb{D}$. Since, q-algebra has four dimensional structure, thus in a four-dimensional theory, a dyon is defined to a particle in which both electric and magnetic charges are taken place. In the high dimensional theories viz. grand unified theory (GUT) and super-string theory (SST) predicted to the existence of both magnetic monopoles and dyons. Thus, a dyon constitutes two four-potentials defined by following quaternionic form [47, 48]:

$$
\begin{aligned}
& \mathbb{A}\left(e_{1}, e_{2}, e_{3}, e_{0}\right)=\left\{A_{x}, A_{y}, A_{z},-\frac{i}{c} \phi_{e}\right\}, \\
& \mathbb{B}\left(e_{1}, e_{2}, e_{3}, e_{0}\right)=\left\{B_{x}, B_{y}, B_{z},-\frac{i}{c} \phi_{m}\right\},
\end{aligned}
$$

where $\mathbb{A}$ and $\mathbb{B}$ are electric and magnetic four-potentials and their real and imaginary parts constituent a generalized potential of dyons, i.e.

$$
\mathbb{V}^{\nu}\left\{A^{\nu}, B^{\nu}\right\}=\left(A^{\nu}+i B^{\nu}\right),(\nu=0,1,2,3) .
$$

As such, the quaternionic form of two four-currents of dyons, respectively,

$$
\begin{aligned}
\mathbb{J}\left(e_{1}, e_{2}, e_{3}, e_{0}\right) & =\left\{J_{x}, J_{y}, J_{z},-i c \rho_{e}\right\}, \\
\mathbb{K}\left(e_{1}, e_{2}, e_{3}, e_{0}\right) & =\left\{K_{x}, K_{y}, K_{z},-i c \rho_{m}\right\},
\end{aligned}
$$

constitute a generalized current-source of dyons. Correspondingly, the quaternionic generalized current defined by

$$
\mathbb{J}^{\nu}\left\{J^{\nu}, K^{\nu}\right\}=\left(J^{\nu}+i K^{\nu}\right) \text {. }
$$

Similarly, the quaternionic representation of energy-momentum four-vector of moving dyons become

$$
\mathbb{P}\left(e_{1}, e_{2}, e_{3}, e_{0}\right)=\left\{p_{x}, p_{y}, p_{z},-\frac{i}{c} E\right\}
$$


Now, we may write the electromagnetic classical field equations called generalized Dirac Maxwell's (GDM) equations for dyons in terms of following symmetric nature,

$$
\begin{aligned}
& \vec{\nabla} \cdot \overrightarrow{\mathcal{E}}+\frac{\partial \Lambda_{e}}{\partial t}-\frac{\rho_{e}}{\varepsilon_{0}}=0, \\
& \vec{\nabla} \cdot \overrightarrow{\mathcal{H}}+\frac{\partial \Lambda_{m}}{\partial t}-\mu_{0} \rho_{m}=0, \\
& \vec{\nabla} \times \overrightarrow{\mathcal{E}}+\frac{\partial \overrightarrow{\mathcal{H}}}{\partial t}+\vec{\nabla} \Lambda_{m}+\mu_{0} \vec{K}=0, \\
& \vec{\nabla} \times \overrightarrow{\mathcal{H}}-\frac{1}{c^{2}} \frac{\partial \overrightarrow{\mathcal{E}}}{\partial t}+\vec{\nabla} \Lambda_{e}-\mu_{0} \vec{J}=0 .
\end{aligned}
$$

where $\overrightarrow{\mathcal{E}} \longmapsto\left(e_{1} \mathcal{E}_{x}, e_{2} \mathcal{E}_{y}, e_{3} \mathcal{E}_{z}\right)$ and $\overrightarrow{\mathcal{H}} \longmapsto\left(e_{1} \mathcal{H}_{x}, e_{2} \mathcal{H}_{y}, e_{3} \mathcal{H}_{z}\right)$ are denoted the quaternionic electric and the magnetic field vectors; $\rho_{e}$ and $\rho_{m}$ are the charge source density due to electric and magnetic charge (monopole); $\varepsilon_{0}$ is the free space permittivity and $\mu_{0}$ is the permeability to the case of free space. Here $\Lambda_{e}$ and $\Lambda_{m}$ are defined the relativistic Lorentz gauge, respectively for electric and magnetic charges of dyons, i.e.

$$
\begin{gathered}
\Lambda_{e} \longmapsto\left[\vec{\nabla} \cdot \vec{A}+\frac{1}{c^{2}} \frac{\partial \phi_{e}}{\partial t}\right]=0, \\
\Lambda_{m} \longmapsto\left[\vec{\nabla} \cdot \vec{B}+\frac{1}{c^{2}} \frac{\partial \phi_{m}}{\partial t}\right]=0 .
\end{gathered}
$$

The GDM equations are invariant under the following duality transformations

$$
\begin{array}{ll}
\overrightarrow{\mathcal{E}} \longmapsto c \overrightarrow{\mathcal{H}}, & \overrightarrow{\mathcal{H}} \longmapsto-\overrightarrow{\mathcal{E}} / c, \\
\vec{J} \longmapsto \vec{K} / c, & \vec{K} \longmapsto-c \vec{J}, \\
\rho_{e} \longmapsto \rho_{m} / c, & \rho_{m} \longmapsto-c \rho_{e} .
\end{array}
$$

These transformations suggested that the duality transformations of magnetic and electric current densities follow those of the electric and magnetic fields, respectively. In GDM equations, the generalized electric and magnetic field vectors can be expressed in terms of two four-potentials of dyons,

$$
\begin{aligned}
& \overrightarrow{\mathcal{E}}=-\vec{\nabla} \phi_{e}-\frac{\partial \vec{A}}{\partial t}-\vec{\nabla} \times \vec{B}, \\
& \overrightarrow{\mathcal{H}}=-\vec{\nabla} \phi_{m}-\frac{\partial \vec{B}}{\partial t}+\vec{\nabla} \times \vec{A} .
\end{aligned}
$$

Now, the quaternionic vector field $\vec{\Omega}$ associated with generalized electromagnetic fields of dyons is defined by

$$
\vec{\Omega}_{\text {Dyon }}=(\overrightarrow{\mathcal{E}}+i \overrightarrow{\mathcal{H}}), i=\sqrt{-1}
$$


The classical fields equations may also be expressed in terms of two four-potentials of dyons, i.e.

$$
\begin{aligned}
\nabla^{2} \vec{A}-\frac{1}{c^{2}} \frac{\partial^{2} \vec{A}}{\partial t^{2}}+\vec{\nabla} \Lambda_{e}+\mu_{0} \vec{J} & =0, \\
\nabla^{2} \vec{B}-\frac{1}{c^{2}} \frac{\partial^{2} \vec{B}}{\partial t^{2}}+\vec{\nabla} \Lambda_{m}-\mu_{0} \vec{K} & =0, \\
\nabla^{2} \phi_{e}-\frac{1}{c^{2}} \frac{\partial^{2} \phi_{e}}{\partial t^{2}}+\frac{\partial \Lambda_{e}}{\partial t}+\frac{\rho_{e}}{\varepsilon_{0}} & =0, \\
\nabla^{2} \phi_{m}-\frac{1}{c^{2}} \frac{\partial^{2} \phi_{m}}{\partial t^{2}}+\frac{\partial \Lambda_{m}}{\partial t}+\mu_{0} \rho_{m} & =0 .
\end{aligned}
$$

These equations are second order differential wave equations, associated with two four-current sources to the case of generalized electromagnetic field of dyons. On the other side, we also may write the electric and magnetic wave equations for dyons in conducting medium,

$$
\begin{gathered}
\frac{1}{c^{2}} \frac{\partial^{2} \overrightarrow{\mathcal{E}}}{\partial t^{2}}-\nabla^{2} \overrightarrow{\mathcal{E}}+\mu_{0} \sigma_{e} \frac{\partial \overrightarrow{\mathcal{E}}}{\partial t}+\vec{\nabla}\left(\frac{\rho_{e}}{\varepsilon_{0}}\right)=0, \\
\frac{1}{c^{2}} \frac{\partial^{2} \overrightarrow{\mathcal{H}}}{\partial t^{2}}-\nabla^{2} \overrightarrow{\mathcal{H}}+\mu_{0} \sigma_{m} \frac{\partial \overrightarrow{\mathcal{H}}}{\partial t}+\vec{\nabla}\left(\mu_{0} \rho_{m}\right)=0 .
\end{gathered}
$$

where $\sigma_{e}$ and $\sigma_{m}$ are the conductivities due to electric charge and magnetic monopole, respectively. In generalized field of dyons, the unified electromagnetic wave equations become

$$
\frac{1}{c^{2}} \frac{\partial^{2} \vec{\Omega}_{\text {Dyon }}}{\partial t^{2}}-\nabla^{2} \vec{\Omega}_{\text {Dyon }}+\mu_{0} \sigma \frac{\partial \vec{\Omega}_{\text {Dyon }}}{\partial t}+\mu_{0} \vec{\nabla} \varrho=0,
$$

where $\sigma\left(\sigma_{e}, \sigma_{m}\right)$ is assumed to the conductivity of dyons and $\varrho \simeq\left(c^{2} \rho_{e}+i \rho_{m}\right)$ is the effective charge density of dyons. We also may write the generalized vector potential wave equations for dyons in free space,

$$
\begin{gathered}
\frac{1}{c^{2}} \frac{\partial^{2} \vec{A}}{\partial t^{2}}-\nabla^{2} \vec{A}+\mu_{0} \sigma_{e} \frac{\partial \vec{A}}{\partial t}+\vec{\nabla} \Lambda_{e}^{C}=0, \\
\frac{1}{c^{2}} \frac{\partial^{2} \vec{B}}{\partial t^{2}}-\nabla^{2} \vec{B}+\mu_{0} \sigma_{m} \frac{\partial \vec{B}}{\partial t}+\vec{\nabla} \Lambda_{m}^{C}=0,
\end{gathered}
$$

and the unified form of wave equations (4.27) and (4.28) become,

$$
\frac{1}{c^{2}} \frac{\partial^{2} \overrightarrow{\mathbb{V}}}{\partial t^{2}}-\nabla^{2} \overrightarrow{\mathbb{V}}+\mu_{0} \sigma_{e, m} \frac{\partial \overrightarrow{\mathbb{V}}}{\partial t}+\vec{\nabla} \Lambda_{e, m}^{C}=0
$$

Here we may identified the Lorentz gauge for conducting medium, respectively for electric and magnetic charge of dyons, i.e., $\Lambda_{e}^{C} \longmapsto\left(\vec{\nabla} \cdot \vec{A}+\frac{1}{c^{2}} \frac{\partial \phi_{e}}{\partial t}+\mu_{0} \sigma_{e} \phi_{e}\right)$ and $\Lambda_{m}^{C} \longmapsto\left(\vec{\nabla} \cdot \vec{B}+\frac{1}{c^{2}} \frac{\partial \phi_{m}}{\partial t}+\mu_{0} \sigma_{m} \phi_{m}\right)$. If we assume the non-conducting media, then the unified vector potential wave equation for dyons may be expressed as $\square \vec{\nabla}=0$ with the Lorentz gauge condition $\left(\Lambda_{e}^{C}, \Lambda_{m}^{C}\right)=\left(\Lambda_{e}, \Lambda_{m}\right)=0$.

\section{Quantized Maxwell equations for dyons}

We already know that the quantization is a process, which deals the transition from a classical understanding of physical phenomena to a newer understanding known as quantum mechanics. In this section, we have demonstrated the quaternionic generalization of the procedure for building quantum electrodynamics from 
classical electrodynamics. Thus we may compare the classical electromagnetic potential wave equations to the generalized quaternionic quantum wave equations and obtain the following equations [30, 31,

$$
\begin{aligned}
& \frac{1}{c^{2}} \frac{\partial^{2} \vec{A}}{\partial t^{2}}-\nabla^{2} \vec{A}+2\left(\frac{m_{0}}{\hbar}\right) \frac{\partial \vec{A}}{\partial t}+\frac{m_{0}^{2} c^{2}}{\hbar^{2}} \vec{A}=0 \\
& \frac{1}{c^{2}} \frac{\partial^{2} \vec{B}}{\partial t^{2}}-\nabla^{2} \vec{B}+2\left(\frac{m_{0}}{\hbar}\right) \frac{\partial \vec{B}}{\partial t}+\frac{m_{0}^{2} c^{2}}{\hbar^{2}} \vec{B}=0
\end{aligned}
$$

These equations are directly governed the quaternionic quantum potential wave equation for dyons, where we have used the following identities:

$$
\begin{aligned}
\Lambda_{e} & \longmapsto\left(\vec{\nabla} \cdot \vec{A}+\frac{1}{c^{2}} \frac{\partial \phi_{e}}{\partial t}\right)=-\frac{2 m_{0} \phi_{e}}{\hbar}, \\
\Lambda_{m} & \longmapsto\left(\vec{\nabla} \cdot \vec{B}+\frac{1}{c^{2}} \frac{\partial \phi_{m}}{\partial t}\right)=-\frac{2 m_{0} \phi_{m}}{\hbar}, \\
\vec{\nabla} \phi_{e} & =-\frac{\partial \vec{A}}{\partial t} \\
\vec{\nabla} \phi_{m} & =-\frac{\partial \vec{B}}{\partial t}
\end{aligned}
$$

and the quantized currents for dyons becomes

$$
\begin{aligned}
& \vec{J}=-\left(\frac{m_{0}^{2} c^{2}}{\mu_{0} \hbar^{2}}\right) \vec{A} . \\
& \vec{K}=-\left(\frac{m_{0}^{2} c^{2}}{\mu_{0} \hbar^{2}}\right) \vec{B} .
\end{aligned}
$$

Now, we may interpreted these quantum equations (5.3)-(5.8) as fellow:

- Equations (5.3) and (5.4) are indicated to a novel equations which show a quantized Lorentz gauge conditions for electric and magnetic potentials of massive dyons. In other words, we may conclude that in quantum formulation of generalized electromagnetic fields of dyons the Lorentz gauge conditions for electric and magnetic potentials have modified as

$$
\begin{aligned}
\vec{\nabla} \cdot \vec{A}+\left(\frac{1}{c^{2}} \frac{\partial}{\partial t}+\frac{2 m_{0}}{\hbar}\right) \phi_{e} & =0 \\
\vec{\nabla} \cdot \vec{B}+\left(\frac{1}{c^{2}} \frac{\partial}{\partial t}+\frac{2 m_{0}}{\hbar}\right) \phi_{m} & =0
\end{aligned}
$$

- Equations (5.5) and (5.6) are described to the strength of longitudinal components of electric and magnetic field vectors, i.e., $\left(\overrightarrow{\mathcal{E}}_{l} \sim-\vec{\nabla} \phi_{e}\right)$ and $\left(\overrightarrow{\mathcal{H}}_{l} \sim-\vec{\nabla} \phi_{m}\right)$.

- Equations (5.7) and (5.8) are described to the quantized electric and magnetic current density of dyons. These quantized current sources are directly depends on the potentials of dyons and also exhibit the quantum analogue of famous London's equation [49] in presence of electric charge and magnetic monopole. 
Furthermore, we can express the following quaternionic quantum wave equations in presence of dual scalar potentials $\left(\phi_{e}, \phi_{m}\right)$ for dyons, i.e.,

$$
\begin{gathered}
\frac{1}{c^{2}} \frac{\partial^{2} \phi_{e}}{\partial t^{2}}-\nabla^{2} \phi_{e}+2\left(\frac{m_{0}}{\hbar}\right) \frac{\partial \phi_{e}}{\partial t}+\frac{m_{0}^{2} c^{2}}{\hbar^{2}} \phi_{e}=0, \\
\frac{1}{c^{2}} \frac{\partial^{2} \phi_{m}}{\partial t^{2}}-\nabla^{2} \phi_{m}+2\left(\frac{m_{0}}{\hbar}\right) \frac{\partial \phi_{m}}{\partial t}+\frac{m_{0}^{2} c^{2}}{\hbar^{2}} \phi_{m}=0
\end{gathered}
$$

where

$$
\begin{gathered}
\rho_{e}=-\left(\frac{m_{0}^{2}}{\mu_{0} \hbar^{2}}\right) \phi_{e}, \\
\rho_{m}=-\left(\frac{m_{0}^{2} c^{2}}{\mu_{0} \hbar^{2}}\right) \phi_{m},
\end{gathered}
$$

are identified the electric and magnetic charge densities of dyons. Equations (5.11) and (5.12) show a unique quantized connection between the charge densities to the corresponding scalar potentials for dyons.

In order to write the quantized Proca-Maxwell like equations for massive dyons in presence of qQM formalism, substituting the quantized transformation condition given by equation (3.10) along with the quantized current sources equations of dyons in generalized Dirac-Maxwell's equation, we establish

$$
\begin{aligned}
& \vec{\nabla} \cdot \overrightarrow{\mathcal{E}}-\frac{2 m_{0}}{\hbar} \frac{\partial \phi_{e}}{\partial t}-\frac{m_{0}^{2} c^{2}}{\hbar^{2}} \phi_{e}=0, \\
& \vec{\nabla} \cdot \overrightarrow{\mathcal{H}}-\frac{2 m_{0}}{\hbar} \frac{\partial \phi_{m}}{\partial t}-\frac{m_{0}^{2} c^{2}}{\hbar^{2}} \phi_{m}=0, \\
& \vec{\nabla} \times \overrightarrow{\mathcal{E}}+\frac{\partial \overrightarrow{\mathcal{H}}}{\partial t}+\frac{m_{0} c^{2}}{\hbar} \overrightarrow{\mathcal{H}}+\frac{2 m_{0}}{\hbar} \frac{\partial \vec{B}}{\partial t}-\frac{m_{0}^{2} c^{2}}{\hbar^{2}} \vec{B}=0, \\
& \vec{\nabla} \times \overrightarrow{\mathcal{H}}-\frac{1}{c^{2}} \frac{\partial \overrightarrow{\mathcal{E}}}{\partial t}-\frac{m_{0}}{\hbar} \overrightarrow{\mathcal{E}}-\frac{2 m_{0}}{\hbar} \frac{\partial \vec{A}}{\partial t}+\frac{m_{0}^{2} c^{2}}{\hbar^{2}} \vec{A}=0 .
\end{aligned}
$$

These equations represent a novel approach to the quantized Maxwell equations for massive particles, called quantized Proca-Maxwell's (QPM) equations for dyons. However, the Proca equation [50 describes to the classical electrodynamics with finite range (or equivalently with a non-zero mass). If we introduce a quantum factor, i.e., $\lambda=\frac{h}{m_{0} c}$, where $\lambda$ is a Compton wavelength for dyons, then the wave propagation vector $(\vec{\kappa})$ of dyons become $\vec{\kappa}=\left(\frac{4 \pi^{2}}{\lambda^{2}}\right)^{1 / 2} \simeq\left(\frac{m_{0}^{2} c^{2}}{\hbar^{2}}\right)^{1 / 2}$. Therefore, we may write the compact QPM equations in terms of wave propagation vector as

$$
\begin{aligned}
\vec{\nabla} \cdot \overrightarrow{\mathcal{E}} & =\frac{2 m_{0}}{\hbar} \frac{\partial \phi_{e}}{\partial t}+\kappa^{2} \phi_{e}, \\
\vec{\nabla} \cdot \overrightarrow{\mathcal{H}} & =\frac{2 m_{0}}{\hbar} \frac{\partial \phi_{m}}{\partial t}+\kappa^{2} \phi_{m}, \\
\vec{\nabla} \times \overrightarrow{\mathcal{E}}+\frac{\partial \overrightarrow{\mathcal{H}}}{\partial t} & =-\frac{m_{0} c^{2}}{\hbar} \overrightarrow{\mathcal{H}}-\frac{2 m_{0}}{\hbar} \frac{\partial \vec{B}}{\partial t}+\kappa^{2} \vec{B}, \\
\vec{\nabla} \times \overrightarrow{\mathcal{H}}-\frac{1}{c^{2}} \frac{\partial \overrightarrow{\mathcal{E}}}{\partial t} & =\frac{m_{0}}{\hbar} \overrightarrow{\mathcal{E}}+\frac{2 m_{0}}{\hbar} \frac{\partial \vec{A}}{\partial t}-\kappa^{2} \vec{A} .
\end{aligned}
$$

Interestingly, these compact QPM equations of massive dyons are invariant under the duality transformations, i.e., $\overrightarrow{\mathcal{E}} \longmapsto c \overrightarrow{\mathcal{H}}, c \overrightarrow{\mathcal{H}} \longmapsto-\overrightarrow{\mathcal{E}}, c \vec{A} \longmapsto \vec{B}, \vec{B} \longmapsto-c \vec{A}, \phi_{e} \longmapsto c \phi_{m}$, and $c \phi_{m} \longmapsto-\phi_{e}$. Thus, the QPM equations are more compact in quaternionic formulation where the scalar coefficient of quaternionic field 
visualized to the scalar electromagnetic field equations and the vector coefficients of q-algebra visualized to the vector electromagnetic field equations of massive dyons. Equations (5.17)-(5.20) can also governed to the massless field equations if $m_{0} \sim 0$, i.e.,

$$
\begin{aligned}
\vec{\nabla} \cdot \overrightarrow{\mathcal{E}} & =0, \\
\vec{\nabla} \cdot \overrightarrow{\mathcal{H}} & =0, \\
\vec{\nabla} \times \overrightarrow{\mathcal{E}}+\frac{\partial \overrightarrow{\mathcal{H}}}{\partial t} & =0, \\
\vec{\nabla} \times \overrightarrow{\mathcal{H}}-\frac{1}{c^{2}} \frac{\partial \overrightarrow{\mathcal{E}}}{\partial t} & =0 .
\end{aligned}
$$

The above equations are generally described to the macroscopic symmetrical Maxwell's equations for massless dyons in free space. In the quaternionic quantum formalism it must be noted that every massive particle will be contained to a current-source in the quantized electromagnetic field of dyons. On the other hand, we can say that quantization of the electromagnetic field of dyons will be occurred only if the particles have involved some finite mass, otherwise it shows classical field equations in free space.

\section{Quantized continuity equations and the wave propagation}

In order to establish the quantized continuity equations for the generalized electromagnetic field of dyons, let us start with the quaternionic quantized Lorentz gauge condition for electric and magnetic charges of dyons given by equation (5.3) and (5.4), i.e.,

$$
\begin{aligned}
& \Lambda_{e}^{\mathfrak{Q}} \longmapsto \quad \vec{\nabla} \cdot \vec{A}+\frac{1}{c^{2}} \frac{\partial \phi_{e}}{\partial t}+\frac{2 m_{0} \phi_{e}}{\hbar}=0, \\
& \Lambda_{m}^{\mathfrak{Q}} \longmapsto \vec{\nabla} \cdot \vec{B}+\frac{1}{c^{2}} \frac{\partial \phi_{m}}{\partial t}+\frac{2 m_{0} \phi_{m}}{\hbar}=0 .
\end{aligned}
$$

As such, we have expressed a relation between the classical and quantum Lorentz gauge conditions of electromagnetic fields for dyons, i.e.,

$$
\begin{aligned}
& \Lambda_{e}^{\mathfrak{Q}}=\Lambda_{e}+\xi \phi_{e}, \\
& \Lambda_{m}^{\mathfrak{Q}}=\Lambda_{m}+\xi \phi_{m} .
\end{aligned}
$$

where the constant $\left(\xi \longmapsto 2 m_{0} / \hbar\right)$ is a quantum term depending upon the mass of the particle. As such, for massless dyons, $\Lambda_{e}^{\mathfrak{Q}} \simeq \Lambda_{e}$ and $\Lambda_{m}^{\mathfrak{Q}} \simeq \Lambda_{m}$. Therefore, we can summarize that the classical theory shows an approximation of the quantum theory. By substitute to the quantized value of four-potentials of dyons, we established the generalized current-source equations for massive dyons as

$$
\begin{aligned}
\vec{\nabla} \cdot \vec{J}+\frac{\partial \rho_{e}}{\partial t}+\xi c^{2} \rho_{e} & =0 . \\
\vec{\nabla} \cdot \vec{K}+\frac{1}{c^{2}} \frac{\partial \rho_{m}}{\partial t}+\xi \rho_{m} & =0 .
\end{aligned}
$$

These are the quantized continuity equations for massive dyons, where the additional third terms visualized the torque density result from the two spin states. Besides, we may obtain the quantized relativistic wave 
propagation of massive dyons by the following manner,

$$
\begin{aligned}
& \left(\square-\vec{\kappa}^{2}\right) \vec{A}=\xi \frac{\partial \vec{A}}{\partial t}, \\
& \left(\square-\vec{\kappa}^{2}\right) \vec{B}=\xi \frac{\partial \vec{B}}{\partial t}, \\
& \left(\square-\vec{\kappa}^{2}\right) \phi_{e}=\xi \frac{\partial \phi_{e}}{\partial t}, \\
& \left(\square-\vec{\kappa}^{2}\right) \phi_{m}=\xi \frac{\partial \phi_{m}}{\partial t} .
\end{aligned}
$$

Here, equations (6.7)-(6.10) are represented the quantized Klein-Gordon (QKG) field equations for massive dyons. Thus, the generalized relativistic QKG wave equations are related to the Schrödinger wave equation in quantum theory. It is second order in space-time and manifestly invariant under the duality transformations. Finally,

$$
\begin{aligned}
& \square \vec{A}=-\mu_{0} \vec{J}+\xi \frac{\partial \vec{A}}{\partial t}, \\
& \square \vec{B}=-\mu_{0} \vec{K}+\xi \frac{\partial \vec{B}}{\partial t}, \\
& \square \phi_{e}=-\frac{\rho_{e}}{\varepsilon_{0}}+\xi \frac{\partial \phi_{e}}{\partial t}, \\
& \square \phi_{m}=-\mu_{0} \rho_{m}+\xi \frac{\partial \phi_{e}}{\partial t},
\end{aligned}
$$

are represented the quantized electromagnetic (QEM) potential wave equations of massive dyons. In fourvectors form, the unified QEM potential wave equation for dyons become

$$
\square \mathbb{V}^{\nu}=-\alpha \mathbb{J}^{\nu}+\xi \frac{\partial \mathbb{V}^{\nu}}{\partial t}
$$

where $\alpha$ is related to electric and magnetic constants. Here, we may see that the main distinguish between classical and quantum formulation is the quantized time varying potential of dyons comes from the small perturbation terms of time scale given by equation (3.10). Therefore, we can summarize the quaternionic representation of classical and the quantum equations for the electromagnetic fields of dyons given by Table- 1 .

\section{Quantized conductivity in electric and magnetic fields of dyons}

In case of quantized electromagnetic fields of dyons in conducting media, we may define the microscopic wave propagation of electric field vector $(\overrightarrow{\mathcal{E}})$ of dyons as

$$
\frac{1}{c^{2}} \frac{\partial^{2} \overrightarrow{\mathcal{E}}}{\partial t^{2}}-\nabla^{2} \overrightarrow{\mathcal{E}}+2\left(\frac{m_{0}}{\hbar}\right) \frac{\partial \overrightarrow{\mathcal{E}}}{\partial t}+\frac{m_{0}^{2} c^{2}}{\hbar^{2}} \overrightarrow{\mathcal{E}}=0
$$


Table 1: Generalized classical and quantum equations of dyons

\begin{tabular}{|c|c|c|}
\hline Dyonic fields & Classical equations $(\xi \sim 0)$ & Quantum equations $\left(\xi \longmapsto 2 m_{0} / \hbar\right)$ \\
\hline $\begin{array}{l}\text { Vector } \\
\text { waves: }\end{array}$ & $\left\{\begin{array}{l}\square \vec{A}=-\mu_{0} \vec{J} \\
\square \vec{B}=-\mu_{0} \vec{K}\end{array}\right.$ & $\left\{\begin{array}{l}\square \vec{A}=-\mu_{0} \vec{J}+\xi \frac{\partial \vec{A}}{\partial t}, \\
\square \vec{B}=-\mu_{0} \vec{K}+\xi \frac{\partial \vec{B}}{\partial t},\end{array}\right.$ \\
\hline $\begin{array}{l}\text { Scalar } \\
\text { waves: }\end{array}$ & $\left\{\begin{array}{l}\square \phi_{e}=-\frac{\rho_{e}}{\varepsilon_{0}}, \\
\square \phi_{m}=-\mu_{0} \rho_{m}\end{array}\right.$ & $\left\{\begin{array}{l}\square \phi_{e}=-\frac{\rho_{e}}{\varepsilon_{0}}+\xi \frac{\partial \phi_{e}}{\partial t}, \\
\square \phi_{m}=-\mu_{0} \rho_{m}+\xi \frac{\partial \phi_{e}}{\partial t},\end{array}\right.$ \\
\hline $\begin{array}{c}\text { Four-Potentials } \\
\text { waves: }\end{array}$ & $\square \mathbb{V}^{\nu}=-\alpha \mathbb{J}^{\nu}$ & $\square \mathbb{V}^{\nu}=-\alpha \mathbb{J}^{\nu}+\xi \frac{\partial \mathbb{V}^{\nu}}{\partial t}$ \\
\hline $\begin{array}{l}\text { Lorentz } \\
\text { gauge: }\end{array}$ & $\begin{array}{l}\vec{\nabla} \cdot \vec{A}+\frac{1}{c^{2}} \frac{\partial \phi_{e}}{\partial t}=0 \\
\vec{\nabla} \cdot \vec{B}+\frac{1}{c^{2}} \frac{\partial \phi_{m}}{\partial t}=0\end{array}$ & $\left\{\begin{array}{l}\vec{\nabla} \cdot \vec{A}+\frac{1}{c^{2}} \frac{\partial \phi_{e}}{\partial t}+\xi \phi_{e}=0 \\
\vec{\nabla} \cdot \vec{B}+\frac{1}{c^{2}} \frac{\partial \phi_{m}}{\partial t}+\xi \phi_{m}=0\end{array}\right.$ \\
\hline $\begin{array}{l}\text { Continuity } \\
\text { equations: }\end{array}$ & $\begin{array}{l}\vec{\nabla} \cdot \vec{J}+\frac{\partial \rho_{e}}{\partial t}=0 \\
\vec{\nabla} \cdot \vec{K}+\frac{1}{c^{2}} \frac{\partial \rho_{m}}{\partial t}=0\end{array}$ & $\left\{\begin{array}{l}\vec{\nabla} \cdot \vec{J}+\frac{\partial \rho_{e}}{\partial t}+\xi c^{2} \rho_{e}=0 \\
\vec{\nabla} \cdot \vec{K}+\frac{1}{c^{2}} \frac{\partial \rho_{m}}{\partial t}+\xi \rho_{m}=0\end{array}\right.$ \\
\hline
\end{tabular}

Here, equation (7.1) can be obtained by comparing the quaternionic wave equation (3.9) to the electric wave equation in conducting medium (4.25). Once, we have obtained the following identities

$$
\begin{aligned}
2\left(\frac{m_{0}}{\hbar}\right) & =\mu_{0} \sigma_{e}, \\
\frac{m_{0}^{2} c^{2}}{\hbar^{2}} \overrightarrow{\mathcal{E}} & =\vec{\nabla}\left(\frac{\rho_{e}}{\varepsilon_{0}}\right) .
\end{aligned}
$$

Thus from equations (7.2) and (7.3), we may governed the following useful relations,

$$
\begin{aligned}
\sigma_{e} & =\frac{2 m_{0}}{\mu_{0} \hbar} \simeq \frac{\xi}{\mu_{0}} \\
\overrightarrow{\mathcal{E}} & =\frac{\hbar^{2}}{\varepsilon_{0} m_{0}^{2} c^{2}}\left(\vec{\nabla} \rho_{e}\right)=-\vec{\nabla} \phi_{e} \sim \overrightarrow{\mathcal{E}}_{l} .
\end{aligned}
$$

Equation (7.4) defines to the quantized electrical conductivity of the electron which is proportional to the mass of the particle, while equation (7.5) express the effective value of quantized electric field vector. Similarly, we may write the microscopic wave equation of magnetic field vector $(\overrightarrow{\mathcal{H}})$ for dyons as

$$
\frac{1}{c^{2}} \frac{\partial^{2} \overrightarrow{\mathcal{H}}}{\partial t^{2}}-\nabla^{2} \overrightarrow{\mathcal{H}}+2\left(\frac{m_{0}}{\hbar}\right) \frac{\partial \overrightarrow{\mathcal{H}}}{\partial t}+\frac{m_{0}^{2} c^{2}}{\hbar^{2}} \overrightarrow{\mathcal{H}}=0
$$

where the quantum identities are governed

$$
\begin{aligned}
2\left(\frac{m_{0}}{\hbar}\right) & =\mu_{0} \sigma_{m}, \\
\frac{m_{0}^{2} c^{2}}{\hbar^{2}} \overrightarrow{\mathcal{H}} & =\vec{\nabla}\left(\mu_{0} \rho_{m}\right) .
\end{aligned}
$$


As such, the magnetic conductivity of magnetic monopole and the effective value of magnetic field vector $(\overrightarrow{\mathcal{H}})$ are expressed as, respectively,

$$
\begin{aligned}
\sigma_{m} & =\frac{2 m_{0}}{\mu_{0} \hbar} \simeq \frac{\xi}{\mu_{0}}=\sigma_{e} \\
\overrightarrow{\mathcal{H}} & =-\vec{\nabla} \phi_{m} \sim \overrightarrow{\mathcal{H}}_{l} .
\end{aligned}
$$

It should be noticed that the value of electric and magnetic conductivity is same to the case of quantized

electric and magnetic fields of dyons. Moreover, we also may govern the following dynamic equations of massive dyons for the case of two-four current-sources, i.e.

$$
\vec{\nabla} \rho_{e}+\frac{1}{c^{2}} \frac{\partial \vec{J}}{\partial t}=0, \quad \vec{\nabla} \rho_{m}+\frac{\partial \vec{K}}{\partial t}=0
$$

where the dynamic equations of massive dyons have been already discussed [51, 52].

\section{Quantized Dirac wave equations for dyons}

Let us start with the relativistic quantum wave equation [53. or Dirac equation for the particles of mass $m_{0}$ can be expressed as

$$
i \hbar \frac{\partial \Psi}{\partial t}=\left[c \vec{\alpha} \cdot(-i \hbar \vec{\nabla})+\beta m_{0} c^{2}\right] \Psi
$$

Here $\vec{\alpha}$ and $\beta$ are four Hermitian matrices defined by following $2 \times 2$ Pauli's $\sigma$-matrices:

$$
\vec{\alpha}=\left(\begin{array}{ll}
0 & \sigma \\
\sigma & 0
\end{array}\right), \beta=\left(\begin{array}{cc}
1 & 0 \\
0 & -1
\end{array}\right),
$$

where,

$$
\alpha_{x}^{2}=\alpha_{y}^{2}=\alpha_{z}^{2}=\beta^{2}=1
$$

and

$$
\begin{aligned}
\alpha_{x} \alpha_{y}+\alpha_{y} \alpha_{x} & =\alpha_{y} \alpha_{z}+\alpha_{z} \alpha_{y}=\alpha_{z} \alpha_{x}+\alpha_{x} \alpha_{z} \\
& =\alpha_{x} \beta+\beta \alpha_{x}=\alpha_{y} \beta+\beta \alpha_{y}=\alpha_{z} \beta+\beta \alpha_{z}=0 .
\end{aligned}
$$

Now, squaring both side of equation (8.1) and obtained

$$
\begin{gathered}
(\vec{\alpha} \cdot \vec{\nabla})^{2} \Psi=\left(-\frac{i m_{0} c}{\hbar} \beta-\frac{1}{c} \frac{\partial}{\partial t}\right)^{2} \Psi \\
\frac{1}{c^{2}} \frac{\partial^{2} \Psi}{\partial t^{2}}-\nabla^{2} \Psi+\frac{2 i m_{0} \beta}{\hbar} \frac{\partial \Psi}{\partial t}-\frac{m_{0}^{2} c^{2}}{\hbar^{2}} \Psi=0 .
\end{gathered}
$$

Interestingly, equation (8.5) can be written in terms of quaternionic QEM wave equation for dyons as

$$
\frac{1}{c^{2}} \frac{\partial^{2} \vec{\Omega}_{\text {Dyon }}}{\partial t^{2}}-\nabla^{2} \vec{\Omega}_{\text {Dyon }}+\frac{2 M_{e f f}}{\hbar} \frac{\partial \vec{\Omega}_{\text {Dyon }}}{\partial t}+\frac{M_{e f f}^{2} c^{2}}{\hbar^{2}} \vec{\Omega}_{\text {Dyon }}=0
$$


where we have used

$$
M_{e f f} \longmapsto\left(i m_{0} \beta\right)
$$

Here the effective mass of dyons $\left(M_{e f f}\right)$ is behave like imaginary mass (like techyonic dyons). The Bogomolny bound [54] shows the effective mass of dyons which is $M_{e f f} \simeq\left(m_{0}^{e}+i m_{0}^{g}\right)$, where $m_{0}^{e}$ is the mass of electron and $m_{0}^{g}$ is the mass of magnetic monopole. According to the GUTs, we can estimate that the mass of monopole [55] can be up to an enormous $10^{17} \mathrm{GeV} / \mathrm{c}^{2}$, which is far greater than the mass of electron $\left(0.511 \mathrm{MeV} / \mathrm{c}^{2}\right)$. Thus we can assumed that the effective mass of dyons become $M_{\text {eff }} \simeq i m_{0}^{g}$.

Now, applying four components spinor of state QEM vector $\vec{\Omega}_{\text {Dyon }}$ associated with two components doublets, i.e., $\vec{\Omega}_{\text {Dyon }} \longmapsto\left(\begin{array}{c}\vec{\Omega}^{+} \\ \vec{\Omega}^{-}\end{array}\right)$. Then the quaternionic quantized Dirac wave equations for dyons can be written as [56, 57,

$$
\begin{aligned}
& \frac{1}{c^{2}} \frac{\partial^{2} \vec{\Omega}^{+}}{\partial t^{2}}-\nabla^{2} \vec{\Omega}^{+}+\frac{2\left(i m_{0} \beta\right)}{\hbar} \frac{\partial \vec{\Omega}^{+}}{\partial t}+\frac{m_{0}^{2} c^{2}}{\hbar^{2}} \vec{\Omega}^{+}=0, \\
& \frac{1}{c^{2}} \frac{\partial^{2} \vec{\Omega}^{-}}{\partial t^{2}}-\nabla^{2} \vec{\Omega}^{-}-\frac{2\left(i m_{0} \beta\right)}{\hbar} \frac{\partial \vec{\Omega}-}{\partial t}+\frac{m_{0}^{2} c^{2}}{\hbar^{2}} \vec{\Omega}^{-}=0 .
\end{aligned}
$$

Thus equations (8.8) and (8.9) are governed two distinguish energy solutions with $\left(E \pm m_{0} c^{2}\right)$ and two possible eigen-states for each of these eigenvalues. Therefore, from these two novel quantum equations we may concluded that there is the existence of antiparticle of dyons called antidyons [58 (i.e. the composition of antiparticle of electron and antiparticle of magnetic monopole). We also may analyze the generalized QEM wave solutions of the Dirac equations for relativistic dyons in free space, in which an interference phenomena will occurred between positive and negative energy states of dyons like as the Zitterbewegung wave equation.

\section{Conclusion}

In this paper, we have extended the formulation of complex algebraic equations in terms of quaternionic quantum equations for massive dyons. We have been established a novel approach to qQM, where we have expressed the various dynamic equations for qQM, viz. the quantum equations for a moving particles, the unified structure of quaternionic quantum wave equation and also the quantized transformation condition for time coordinate. The quaternionic form of quantized potential wave equations for dyons has been investigated. The qQM formalism described a novel approach to the Lorentz gauge conditions, respectively, for the electric charge and magnetic monopoles have also been established. We have obtained the quantized current sources equations which are directly depend on the potentials of massive dyons those governed the quantum analogue of famous London's equation in presence of dyons. From the qQM formalism, we also have been found a connection between the quantized electric charge density with the electric scalar potential and quantized magnetic charge density with the magnetic scalar potential of dyons. A new form of quantized Proca-Maxwell equations for dyons has been proposed, which are manifestly invariant under the duality transformations. Furthermore, we have discussed that the quaternionic quantization of the electromagnetic field of dyons will be occurred only if the particles have involved some finite mass, otherwise it shows the classical field equations in free space. Accordingly, the quantized continuity equations for generalized electromagnetic field of massive dyons has been obtained. We have established the quantized Klein-Gordon like field equations and the unified QEM potential wave equation for massive dyons. Moreover, the effective quantized electric and magnetic 
fields vector has been proposed in a conducting medium of massive dyons. Finally, we have been investigated the quaternionic quantized relativistic Dirac wave equations for massive dyons, which indicated that there will be the existence of antiparticle of dyons called antidyons. In many theories related to high energy physics suggested that the dark matter field would be as a source of antiparticles. Rather, in experimental point of view, it is clear that, like magnetic monopoles the dyons and antidyons would be too heavy to be produced at the Large Hadron Collider (LHC). Recently, the experimental study of magnetic monopole (which created dyons) has been proposed by Daviau et. al [59]. Therefore, we may conclude that, from these generalized qQM formulation, it will be easy option to establish the existence of quantum theory for particles viz. dyons, tachyons, axions, weakly interacting massive particles (WIMPs), etc.

\section{References}

[1] P. A. M. Dirac, "Quantized Singularities in the Electromagnetic Field", Proc. Roy. Soc. London, A133 (1931), 60.

[2] P. A. M. Dirac, "The theory of magnetic poles", Phy. Rev., 74 (1948), 817

[3] P. A. M. Dirac, “Generalized Hamiltonion Dynamics", Can. J. Math., 2 (1950), 129.

[4] J. Tamm, "Die verallgemeinerten kugelfunktionen und die wellenfunktionen eines elektrons un felde eines magnetpoles", Z. Phys. 71 (1931), 141.

[5] B. O. Gronblom, "Über singuläre magnet-pole", Ann. Phys., 5 (1938), 32.

[6] D. Sivers, "Possible binding of a magnetic monopole to a particle with electric charge and a magnetic dipole moment", Phys. Rev., D2 (1970), 2048.

[7] H. A. Wilson, "Note on Dirac's Theory of Magnetic Poles", Phys. Rev., 75 (1949), 309.

[8] N. F. Ramsay, "Time Reversal, Charge Conjugation, Magnetic Pole Conjugation, and Parity", Phys. Rev., 109 (1958), 225.

[9] N. Cabibbo and E. Ferrari, "Quantum electrodynamics with Dirac monopoles", Nuovo Cim., 23 (1962), 1147.

[10] S. Weinberg, "Photons and Gravitons in Perturbation Theory: Derivation of Maxwell's and Einstein's Equations", Phys. Rev., 138B (1965), 988.

[11] J. Schwinger, "Magnetic charge and Quantum Field Theory", Phys. Rev., 144 (1966), 1087.

[12] J. Schwinger, "Sources and Magnetic charge," Phys. Rev., 173 (1968), 1536.

[13] J. Schwinger, “A Magnetic Model of Matter", Science, 165 (1969), 757.

[14] D. Zwanziger, "Dirac Magnetic Poles Forbidden in S-Matrix Theory", Phys. Rev., 137B (1965), 647.

[15] A. Peres, "Rotational invariance of magnetic monopoles", Phys. Rev., 167 (1968), 1449.

[16] A. Peres, "Singular String of Magnetic Monopoles", Phys. Rev. Lett., 18 (1967), 50. 
[17] T. M. Van, "Classical theory of magnetic charge", Phys. Rev., 160 (1967), 1182.

[18] I. Bialynicki-birula, "Photon as a Quantum Particle", Acta Phys. Pol. B, 37 (2006), 935.

[19] I. Bialynicki-birula, "On the Wave Function of the Photon", Acta Phys. Pol. A 86 (1994), 97.

[20] J. E. Sipe, "Photon Wave Functions", Phys. Rev. A 52 (1995), 1875.

[21] B. S. Rajput, "Unification of generalized electromagnetic and gravitational fields", J. Math. Phys. 25 (1984), 351.

[22] D. Yerchuck, A. Dovlatova and A. Alexandrov "Classical \& Quantum electrodynamics concept based on Maxwell equation symmetry", arXiv: 1102.2619v4 [math-ph] (2013).

[23] I. V. Polubarinov "Equations of Quantum Electrodynamics", Phys. Particle Nucl., 34 (2003), 377.

[24] B. C. Chanyal, P. S. Bisht and O. P. S. Negi, "Generalized Octonion Electrodynamics", Int. J. Theor. Phys., 49 (2010), 1333.

[25] B. C. Chanyal, S. K. Chanyal, Ö. Bektaş and S. Yüce, "A new approach on electromagnetism with dual number coefficient octonion algebra", Int. J. Geom. Meth. Mod. Phys., 13 (2016), 1630013.

[26] B. C. Chanyal, P. S. Bisht and O. P. S. Negi, "Octonion and conservation laws for dyons", Int. J. Mod. Phys. A, 28 (2013), 1350125.

[27] B. C. Chanyal, "Classical Geometrodynamics with Zorn Vector Matrix Algebra for GravitoDyons", Reports Math. Phys., 76 (2015), 1.

[28] B. C. Chanyal, "Split octonion reformulation of generalized linear gravitational field equations", J. Math. Phys., 56 (2015), 051702.

[29] B. C. Chanyal, V. K. Sharma and O. P. S. Negi, "Octonionic Gravi- Electromagnetism and Dark Matter", Int. J. Theor. Phys., 54 (2015), 3516.

[30] A. I. Arbab, "A Quaternionic Quantum Mechanics," Applied Physics Research, 3 (2011), 160.

[31] A. I. Arbab, "Quantized Maxwell's equations", Optik, 136 (2017) 64.

[32] S. Demir, S. Kekec, "Octonic Massive Field Equations", Int. J. Theor. Phys., 55 (2016), 3338.

[33] S. Demir, M. Tanisli, M. E. Kansu, "Octonic massless field equations”, Int. J. Mod. Phys. A, 30 (2015), 1550084.

[34] S. Demir, M. Tanisli, M. E. Kansu, "Generalized Hyperbolic Octonion Formulation for the Fields of Massive Dyons and Gravito Dyons", Int. J. Theor. Phys. 52 (2013), 3696.

[35] M. Tanisli, M. E. Kansu, S. Demir, "Supersymmetric Quantum Mechanics And Euclidean Dirac Operator With Complexified Quaternions". Mod. Phys. Lett. A, 28 (2013), 1350026.

[36] T. Tolan, M. Tanisli, S. Demir, “Octonic Form of Proca Maxwell's Equations and Relativistic Derivation of Electromagnetism", Int. J. Theor. Phys. 52 (2013), 4488.

[37] S. Demir, M. Tanisli, "Sedenionic Formulation for Generalized Fields of Dyons", Int. J. Theor. Phys. 51 (2012), 1239. 
[38] S. Demir, M. Tanisli, "A compact biquaternionic formulation of massive field equations in gravi electromagnetism", Eur. Phys. J. Plus, 126 (2011), 115.

[39] S. Demir, M. Tanışlı, N. Şahin, M.E. Kansu, "Biquaternionic reformulation of multifluid plasma equations" Chinese J. Phys., 55 (2017), 1329.

[40] B. C. Chanyal, "Sedenion unifed theory of gravi-electromagnetism", Indian J. Phys. 88 1197, (2014).

[41] B. C. Chanyal, "Split Octonion Reformulation for Electromagnetic Chiral Media of Massive Dyons", Commun. Theor. Phys. 68 (2017), 701.

[42] B. C. Chanyal, "A relativistic quantum theory of dyons wave propagation", Canadian J. Phys., 95 (2017), 1200.

[43] W. R. Hamilton, "Elements of quaternions", Chelsea Publications Co., NY, (1969).

[44] P. G. Tait, "An elementary Treatise on Quaternions", Oxford Univ. Press, NY, (1875).

[45] A. I. Arbab, "The analogy between matter and electromagnetic waves", EPL, 94 (2011), 50005.

[46] A. I. Arbab, "Extended electrodynamics and its consequences", Mod. Phys. Lett. B 31 (2017), 1750099 .

[47] H. Dehnen and O. P. S. Negi, "Electromagnetic Duality, Quaternion and Supersymmetric Gauge Theories of Dyons", Pre-print (2006) arXiv:hep-th/0608164.

[48] J. D. Jackson, "Classical Electrodynamics", John Wiley \& Sons, NY, (1962).

[49] F. London, H. London, "The electromagnetic equations of the supraconductor", Proc. R. Soc., A 149 (1935), 71.

[50] A. Proca, "Sur 1'equation de Dirac", Compt. Rend. 190 (1930), 1377.

[51] B. C. Chanyal, "Generalized Klein-Gordon field equations with octonion space-time (OST) algebra", Chinese J. Phys. 55, (2017), 432.

[52] B. C. Chanyal, "Dual octonion electrodynamics with the massive field of dyons", J. Math. Phys., 57 (2016), 033503.

[53] A. Wachter, "Relativistic Quantum Mechanics", Springer International Publishing, Netherlands (2011).

[54] E. B. Bogomolny, "Stability of Classical Solutions”, Sov. J. Nucl. Phys., 24 (1976), 449.

[55] A. Rajantie, "The search for magnetic monopoles", Phys. Today, 69 (2016), 40.

[56] A. I. Arbab, “A New Wave Equation of the Electron”, J. Mod. Phys. 2 (2011), 1012.

[57] A. I. Arbab, "Derivation of Dirac, Klein-Gordon, Schrödinger, diffusion and quantum heat transport equations from a universal quantum wave equation", EPL 92 (2010), 40001.

[58] R. Larsen and E. Shuryak, "Classical interactions of the instanton-dyons with antidyons", Nucl. Phys. A 950 (2016), 110. 
[59] C. Daviau, D. Priem, and G. Racineux, "Tracks of magnetic monopoles", Annales de la Fondation Louis de Broglie 38 (2013), 1. 